\title{
Preparing Australian nurse educators to teach international students
}

\author{
Joanne T. Joyce-McCoach, Dominique R. Parrish \\ School of Nursing, Midwifery and Indigenous Health, University of Wollongong, Wollongong, Australia. \\ Correspondence: Joanne T. Joyce-McCoach. Address: School of Nursing, Midwifery and Indigenous Health, University of \\ Wollongong, Wollongong, Australia. Email: jjoyce@uow.edu.au
}

Received: June 23, 2014

Accepted: August 8, 2014

Online Published: August 21, 2014

DOI : $10.5430 /$ jnep.v4n10p109

URL: http://dx.doi.org/10.5430/jnep.v4n10p109

\begin{abstract}
Australia is the third largest provider of international education, however, the preparedness of academics, teaching international students, may not have kept abreast with the growth in universities' student enrolments in transnational teaching programs. The differences in Western and Asian cultures present challenges that can have a significant impact on the education of international students particularly in transnational teaching programs. As such, there is a need for educators to develop specific strategies to ensure positive student experience, learning and outcomes for international students. A design-based research methodology was adopted to construct, evaluate and endorse a professional development workshop that integrated reflective practice as the method of delivery. The professional development workshop sought primarily to assist nurse educators gain a deeper awareness of the cultural considerations associated with teaching international students and thereby develop strategies to effectively teach this cohort of students. Overall, the participants rated the workshop as highly effective, recognizing the relevance and appropriateness of the workshop content to their professional development needs and for clarifying appropriate and relevant strategies that could be employed when teaching international students. The professional development workshop participants affirmed that the workshop assisted them in developing understanding about cultural factors specific to a cohort of students they taught in Hong Kong. Further, these teachers believed that the integration of identified culturally sensitive teaching strategies in their practice was a direct result of their engagement in the workshops.
\end{abstract}

\section{Key words}

Student experience, Engagement, International students, Reflective teaching approach, Professional development, Nurse educators

\section{I ntroduction}

Australia is the third largest provider of international education with overseas students comprising over 18 percent of the total student enrollment in Australian universities ${ }^{[1,2]}$. While Australian curricula is designed to promote global perspectives, there are educational ideologies that need to be considered when teaching students from different cultural backgrounds, to ensure positive student outcomes and experiences. A substantial body of evidence has stressed the importance of addressing the cultural sensitivities associated with teaching international students. Research affirms the 
need for educators to challenge traditional approaches to teaching and identify culturally appropriate pedagogy to ensure the success of programs delivered to international students ${ }^{[3-6]}$.

The preparedness of academics, teaching international students, has not necessarily kept abreast with the growth in universities' international student enrollments ${ }^{[1,2,7,8]}$. There is a perpetual focus across all Australian universities to ensure the quality of academic's teaching and ultimately student's learning. Almost every, if not all universities in Australia offer formal and informal professional development activities that are designed to enhance academics' teaching and thereby increase the likelihood of positive student experiences and outcomes. Whilst these programs, (e.g. Foundational University Teaching and Learning courses, Graduate Certificate in Higher Education) address higher education teaching and learning broadly they do not necessarily attend to the specific idiosyncrasies associated with teaching international students. This gap was addressed by the initiative described in this paper which sought to design, evaluate and endorse a professional development workshop, to assist nurse educators gain a deeper awareness of the cultural considerations associated with teaching international students and thereby develop strategies to effectively teach this cohort of students. The workshop was implemented in an Australian school of nursing, with academics who were teaching international students in a Master of Nursing program, delivered in Hong Kong (herein referred to as the offshore program). The workshop utilized a reflective learning approach as the key pedagogical strategy to assist participating nurse educators identify factors that could potentially impact on the learning experiences and achievement of students in the offshore program. In light of these judgments, the nurse educators ascertained teaching strategies that they perceived could be integrated into their teaching of international students to positively influence student learning outcomes and experience.

The research findings ensuing from this initiative affirmed the affordances of reflective practice as a means of professionally developing nurse educators. The reflective approach was acknowledged as a highly effective professional development strategy that assisted nurse educators gain a deeper awareness of the cultural considerations associated with teaching international students. Additionally, the participating nurse educators noted that as a consequence of their engagement in the workshop they had greater awareness of and more confidence in their ability to implement strategies to effectively teach students in the offshore program.

\section{Literature}

Reflective practice is well established as an important trait for many practice-based professions, a desirable tertiary graduate quality and a fundamental competency of nursing professionals (Brookfield 2012). Being reflective is increasingly recognized as a highly appropriate and significant strategy in nurse education. Subsequently, skills in reflection are developed as a core component in many undergraduate and postgraduate nursing programs ${ }^{[9,10]}$. Theorists describe reflection as an active and purposeful cognitive process which juxtaposes individual beliefs and knowledge with evidence to inform practice development ${ }^{[11,12]}$. Reflective practice generally addresses real-world problems, allowing for uncertainty and perplexity for the specific objective of identifying solutions to improve practice ${ }^{[9,13]}$.

The differences in western and Asian cultures present challenges that can have a significant impact on student learning ${ }^{[3,5,6]}$. These challenges include but are not limited to issues associated with English being the student's second language, the facilitative delivery style of western teachers and the use of generic, non-discipline specific resources ${ }^{[2,14-16]}$. While these cultural challenges can hinder a students' learning, progress and achievement, they can also be appropriately addressed and their consequent impact mitigated. The literature emphasizes the fact that the cultural background of the student underpins their perception and approach to learning and that recognition of students' identity is an essential first step in teaching international students and addressing cultural challenges ${ }^{[2,4,5,17]}$. The imperative is for educators to develop appropriate and relevant strategies that are responsive to the students' culture and promotes positive student learning and outcomes ${ }^{[1,8,18]}$. The initiative described in this paper sought to design, evaluate and endorse a professional development workshop to assist nurse educators gain a deeper awareness of the cultural background and approach to learning of international students and thereby develop appropriate and relevant strategies to effectively teach this cohort of students. 
Research has established that a university teacher's planned approach to student learning will determine their actual practice in the classroom ${ }^{[1,8,19]}$. If a teacher is going to enhance or develop their classroom practice they must first consider the learning context and the nuances of that context. Subsequently, they need to identify and implement appropriate teaching strategies in light of these considerations. Reflection is not only a sound strategy for considering the learning context but also for strategizing how student learning might effectively be facilitated. By compelling educators to question their practice and contemplate the relevance of teaching strategies they adopt, the potential for new learning and the development of their future practice is enhanced ${ }^{[11,13]}$.

There is minimal evidence to suggest that reflective practice has previously been used in relation to the professional development of nurse educators or even more broadly academics. This investigation and report on the utilization of reflective practice as a conduit of professional development, provides a perspective on this innovative approach for enhancing the pedagogical practices of educators that could inform other academic development initiatives.

\section{Methodology}

Design-based research was the theoretical framework underpinning the approach ${ }^{[20]}$ adopted for this initiative, which sought to construct, evaluate and endorse a professional development workshop, to assist academics to understand the cultural considerations associated with teaching international students and consequently develop culturally appropriate and relevant teaching strategies. Reeves, Herrington and Oliver ${ }^{[20]}$ (see Figure 1) suggest that a design-based research methodology is commonly used and appropriate for investigating and addressing an educational problem. This approach focuses on: assessing the factors impacting on the educational issue or problem; identifying a solution to the issue/problem; implementing, evaluating and further refining the solution to generate an eventual sustainable outcome, which will be implemented or integrated in the future educational practice. Given these affordances of design based research, it was decided that this methodological approach was appropriate to investigate and identify an effective professional development workshop program that would have a positive impact on the teaching practice of nurse educators in the offshore program.

This initiative was conducted as a research project but local research ethics committee guidelines deemed it unnecessary to seek ethical approval because the review did not involve patients and was undertaken as part of the institutional educational review cycle. However ethical principles of confidentiality and anonymity were discussed with respondents and adhered to throughout the project.

The explicit method adopted in this initiative is described in the following sections.

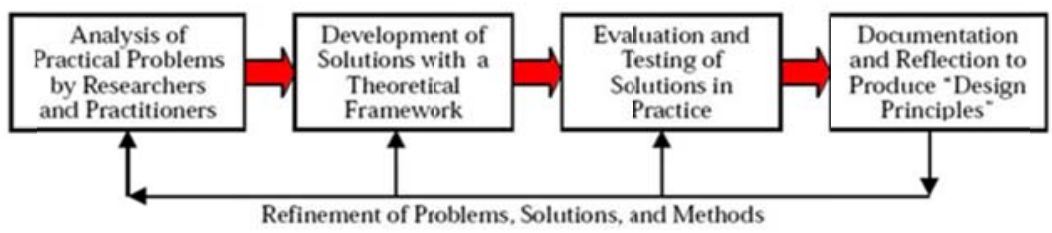

Figure 1. The design based research methodology (Adapted from Reeves, Herrington \& Oliver 2005)

\subsection{Phase 1: Analysis of practical problems by researchers and practitioners}

The initial phase of this Initiative involved an analysis of the programs that had being implemented to professionally develop academics teaching in the offshore program. This analysis was informed by historical records of the previously implemented professional development workshops, including professional development workshop evaluations and the anecdotal observations of the offshore program coordinator.The professional development workshops were conducted annually for the nurse educators teaching in the offshore program, to ensure their preparedness to teach in the offshore 
program. It is important to note that that the nurse educators attending these annual workshops had varying degrees of experience and expertise in regard to teaching international students. This meant that the program that was eventually designed and implemented needed to accommodate these varying degrees of academics' practice and capability. It was also recognized that the workshop should model sound andragogy and as such needed to be participant-centered and engaging. Previous workshop evaluations had criticized the didactic presentation format that was commonly adopted in earlier workshops.

\subsection{Phase 2: Development of solutions with a theoretical framework}

A cyclical process of reviewing the historical records and anecdotal data as well as literature describing and providing examples of effective approaches for professionally developing nurse educators was undertaken to inform the design of the professional development workshop. A range of implementation strategies were extensively contemplated, with a workshop program underpinned by reflective learning subsequently designed. The resultant workshop program integrated engaging participant activities that encouraged the nurse educators to envision opportunities and specific strategies to enhance their teaching practices, particularly for the offshore program context. A strategic decision was made that the professional development workshop would be facilitated by an external consultant to enable the program coordinator to more actively engage in the workshop. The professional development workshop was facilitated prior to the commencement of the first semester of teaching in 2011. Of the fifteen nurse educator staff, teaching in the offshore program, eight attended the professional development workshop. In addition, seven support staff, involved in the delivery of the offshore program, also participated in the workshop.

\subsection{Phase 3: Evaluation and testing of solutions in practice}

The professional development workshop program was evaluated by the participants, with feedback gathered to establish their views of the delivered workshop and ideas for future program enhancements. An evaluation questionnaire, consisting of ten quantitative and five qualitative survey questions was completed by ten of the fifteen workshop participants. A five point Likert scale ranging from (5) strongly agree to (1) strongly disagree, was used in the quantitative questions. In addition, semi-structured interview were conducted with the nurse educators to assess the value and relevance of the workshop for their teaching in the offshore program at the end of Semester 1, 2011. Four of the nurse educators who attended the professional development workshop were interviewed. The interview discussions were summarized and the records were sent to the interviewed nurse educators for validation. The gathered data was analyzed, including thematic analysis of the qualitative data. The quantitative results were used to gauge the perceived appropriateness and value of the professional development workshop. Both the quantitative and qualitative results were used to validate the highly rated aspects of the workshop and adopted approach as a means of effectively developing nurse educators as well as identify design and delivery modifications for future professional development workshop iterations.

\subsection{Phase 4: Reflection and documentation to produce "Design principles"}

The findings from the 'evaluation and testing phase of solutions in practice' were reviewed and subsequently informed the rationalisation of the professional development workshop for nurse educators in the offshore program. This finalised workshop program was delivered to and re-evaluated by nurse educators in 2012 and 2013 with both cohorts affirming and praising the content and reflective learning delivery approach of the workshop.

\subsection{Limitations}

This research investigation was subject to the usual limitations associated with a small sample. This includes the ability to generalise the findings to other contexts, and the subjectivity of the individuals in the small sample. However, the project provides robust evaluation of a professional development initiative that could inform academic development approaches more broadly and compelling support for the utilisation of reflective learning in professional development workshops. The reader is able to generalise the findings from this research initiative to their own contexts where applicable. 


\section{Results}

\subsection{Workshop evaluation data}

The professional development workshop participants included both nurse educators and support staff involved in the delivery of the offshore program. Of the 15 workshop participants, $67 \%(n=10)$ completed a workshop evaluation. The responses from these participants were used to gauge the perceived appropriateness and value of the professional development workshop.

All of the respondents $100 \%(n=10)$, believed that the workshop was well organised, met their expectations, provided them with opportunities to contribute and consolidated their understanding of the cultural considerations that should be contemplated when teaching international students. All of the respondents, $100 \%(\mathrm{n}=10)$ also acknowledged that their contributions were facilitated effectively, time was efficiently managed and the workshop extended their awareness of good practices for engaging international students and addressing cultural considerations. Finally, $80 \%(n=8)$ of the respondents acknowledged the relevance and appropriateness of the workshop content to their professional development needs and for identifying strategies that could be employed when teaching international students. Table 1 presents a summary of the workshop evaluation data.

Table 1. Workshop Evaluation Questionnaire Results

\begin{tabular}{|c|c|c|c|}
\hline & $\mathbf{N}$ & Mean & S.D. \\
\hline 1. The workshop met my expectations. & 10 & 4.8 & 0.4216 \\
\hline $\begin{array}{l}\text { 2. The workshop was relevant to my professional development needs as a subject/unit } \\
\text { coordinator. }\end{array}$ & 8 & 4.625 & 0.5175 \\
\hline $\begin{array}{l}\text { 3. The workshop content was appropriate for clarifying what I can/will do as a teacher in } \\
\text { the offshore program. }\end{array}$ & 8 & 4.625 & 0.5175 \\
\hline $\begin{array}{l}\text { 4. The workshop consolidated my understanding of the cultural considerations that should } \\
\text { be contemplated when teaching students in the offshore program. }\end{array}$ & 10 & 4.8 & 0.4216 \\
\hline $\begin{array}{l}\text { 5. The workshop extended my awareness of good practices for engaging students in the } \\
\text { offshore program and addressing cultural considerations. }\end{array}$ & 10 & 4.8 & 0.4216 \\
\hline $\begin{array}{l}\text { 6. There were adequate opportunities to discuss issues and questions I had in regard to my } \\
\text { role as a teacher in the offshore program. }\end{array}$ & 8 & 4.625 & 0.5175 \\
\hline 7. There were adequate opportunities for me to contribute. & 10 & 4.9 & 0.3162 \\
\hline 8. The contributions of the participants were facilitated effectively. & 10 & 4.8 & 0.4216 \\
\hline 9. Time was managed effectively. & 10 & 4.3 & 0.9487 \\
\hline 10. The workshop was well organized and administered & 10 & 5 & 0 \\
\hline
\end{tabular}

Qualitative findings supported the quantitative results with very positive comments being received about the workshop design and implementation. Thematic analysis of the qualitative data, suggested that participants recognized that the workshop prompted their deeper consideration of strategies for enhancing their current teaching practices and promoting international student engagement and success. Comments that reflected this included:

I appreciate the need to actively support students and attempt to engage with them to ensure that they are aware of my expectations. (Workshop participant \#1)

I will be more explicit in regard to assessment tasks, especially in the use of journal articles. I will develop culturally appropriate examples allowing students to provide their context specific scenarios. (Workshop participant \#5)

I can see the need to develop more online learning support resources that are designed specifically for overseas students. (Workshop participant \#9) 
I am going to consider strategies to enhance understanding of content for international students. (Workshop participant \#10)

The workshop participants were satisfied with the opportunities provided for them to discuss, with their colleagues, issues and questions in regard to their role as a teacher in the offshore program. Acknowledgement of the value of learning from the experiences of others was also strongly communicated in qualitative comments such as:

It was beneficial to have interaction with others; it brought about a Universalization of ideas/thoughts/feelings. (Workshop participant \#2)

Hearing about people's experiences and the cultural tips from various participants was helpful. (Workshop participant \#3)

The workshop being facilitated so all persons could contribute to was a good idea. (Workshop participant \#7)

Very interesting, great to network and hear of colleagues' experiences and stories. Will maintain networking developed today to support my role. (Workshop participant \#9)

\subsection{Workshop impact}

At the conclusion of the semester, the workshop participants who had taught in the offshore program were interviewed. Semi-structured interviews were conducted with all of the academics involved in delivering the Autumn (Semester 1) 2011 offshore program. The thematic analysis of the interview notes focused on evaluating and gauging the impact the professional development workshop had on the teaching practice of the nurse educators in the offshore program. The interviewed academics reaffirmed the value of the workshops and indicated that their awareness of the cultural considerations pertinent to teaching in an international setting had increased as a direct result of the professional development workshop they attended. Further, these academics described how they increasingly used specific strategies, conceptualized during the workshops, in their formal teaching sessions. A key factor in the integration of new and varied teaching approaches was the academics strengthened confidence resulting from a better understanding of the student experience and effective strategies for teaching international students. Nurse educators' assessment of the impact of the professional development workshop was that it enhanced their preparedness for practice by increasing their capability and confidence.

\section{Discussion}

The professional development workshop developed, assessed and refined in this project sought primarily to assist nurse educators gain a deeper awareness of the cultural considerations associated with teaching international students and thereby develop strategies to effectively teach this cohort of students. Feedback from workshop participants affirmed that the workshop consolidated their understanding of the cultural considerations that should be contemplated when teaching international students. Additionally, participants noted that knowledge acquired through the workshop, extended their awareness of effective teaching practices for engaging international students and addressing cultural considerations. Workshop participants also recognized that the approach adopted in facilitating the workshop program was a sound conduit for the development of these knowledge and skills. Nurse academics, who attended the workshop and subsequently delivered the offshore program, affirmed the value and positive impact of the workshop on their teaching practice. These academics acknowledged their heightened awareness of strategies to address cultural considerations when teaching international students. This knowledge, it was reinforced, better prepared the nurse educators for teaching international students. The workshops also enhanced the confidence of the nurse educators which manifested in these academics using effective pedagogical practices for teaching international students. Moreover, the reflective practice approach used to professionally develop the nurse educators identified three benefits. Firstly, the workshop design and delivery integrated and modelled sound pedagogical principles that achieved desired outcomes. Secondly, the workshop 
promoted nurse educators' understanding and awareness of cultural considerations pertinent to teaching international students. Thirdly, as a consequence of their engagement in the workshop the nurse educators' practice was developed.

\subsection{The affordances of reflective practice as an approach for sound professional development}

The professional development workshop and delivery integrated sound pedagogical principles that promoted the practice development of nurse educators. The reflective practice approach catered to the diversity of workshop participants. Individuals were able to engage meaningfully in the workshop activities regardless of their level of experience, expertise or knowledge. The spectrum of academics attending the workshop ranged from those highly experienced in teaching international students offshore, to, and including, those who had never taught in the international program but had aspirations to do so. The reflective approach adopted in the facilitation of the workshops, supported the collaboration of participants and promoted each individual's advancement of knowledge from their own perspective and level of understanding.

The workshop program purposely harnessed the experience and knowledge of those nurse educators who had taught in the offshore program. Reflective activities were facilitated to promote peer learning and the active engagement of all participants. Small group activities focussed on brainstorming potential barriers to learning that may be experienced by international students with subsequent group activities conceptualizing strategies to address these identified barriers. The nature of the activities facilitated in the workshop program were underpinned by authentic learning principles and particularly focussed on the real world relevance and solution focused elements of this learning design.

Workshop evaluation data noted that the use of reflection to explore cultural considerations provided opportunities to develop and consolidate understandings. Feedback also indicated that the workshop program enabled opportunities for participants to learn from the experiences of their peers. Further, participants recognised the workshop content was both relevant and appropriate to their professional needs and practice development. The workshop approach was acknowledged as engaging, collaborative and superior to a didactic information focussed workshop design. The literature echoes these findings noting that when educators question their practice and critically contemplate the relevance of this practice they are likely to identify, adopt and advance their practices ${ }^{[11,13,18]}$.

\subsection{Reflective practice promotes better understanding and deeper awareness}

Feedback from workshop participants acknowledged that the reflective approach developed a deeper understanding of the cultural considerations that should be contemplated when teaching international students and potential strategies that could be adopted to address these considerations. The professional development workshop initially required individuals to identify the cultural considerations they believed fundamental for teaching in the offshore program. This was facilitated firstly, by participants journaling their reflections about what they perceived to be the pertinent factors influencing international students' learning. Following on from this, the workshop participants interrogated their reflections in small groups, to establish a collective summary of key cultural considerations for teaching international students. The summaries were then discussed in the small groups and strategies to address the identified challenges were brainstormed before being shared with the larger group. It was evident from this preliminary activity that the workshop participants had a range of insights into the cultural considerations and associated strategies pertinent for teaching international students. As a consequence of this reflective activity, the perceptions of individuals were augmented and a comprehensive suite of sound pedagogical practices identified. Confirmation of the value of these workshop activities was evidenced in the comment:

Discussion with people who have been involved in the program was invaluable. (Workshop participant \#1)

This process of reflective thinking and identification of solutions to improve practice is reinforced in the literature ${ }^{[9,11,13]}$. The two elements of the workshop design that were acknowledged as most effective were firstly, that participants had the opportunity to extensively explore cultural nuances specific to engaging international students in the offshore program. 
Secondly, participants affirmed that the approach extended their thinking past superficial stereotypes generally associated with international students to an increased level of cultural sensitivity. This resulted in the enhanced cultural awareness of the participants and the establishment of specific strategies to address the identified cultural considerations, as summed up in the comment:

I felt that I had learnt a lot about the offshore program during the workshop and the barriers associated with learning and teaching in Hong Kong; this lead to my identifying strategies to overcome potential adversities and facilitate sound teaching strategies. (Nurse Educator \#3)

Reflection, undertaken in the workshop, assisted the participants to question their practice and contemplate the relevance of teaching strategies adopted in the offshore program. The potential for new learning and the development of future practice was facilitated by the reflection activity. Johns ${ }^{[13]}$ supports the importance of reflection in achieving such outcomes. The approach enabled all of the participants to actively engage in the learning activities and make meaningful contributions, a benefit of the reflective approach that was highlighted in the comment:

Getting participants to contribute to the ideas/workshop really improved the level of their interaction. The workshop was a good activity for exploring issues and solutions. (Workshop participant \#8)

\subsection{Reflection can influence a change in practice and increased confidence}

Academics that participated in the professional development workshop and were involved in the offshore program delivery in Autumn (Semester 1) 2011 confirmed, in the practitioner interviews, that they had used knowledge and skills gained through the workshop, in their offshore teaching. Further, it was noted that the workshop enhanced nurse educators' confidence both to integrate acquired knowledge and skills into teaching practice and contemplate teaching in the offshore program. This was reinforced in the comments:

I have used strategies discussed in the workshop within my teaching in Hong Kong. I felt better prepared and more confident in my teaching. (Nurse Educator \#2)

Drawing on the experience of staff that have faced some challenges and how they resolved these made teaching in the offshore program seem less daunting. (Workshop participant \#8)

Research has established that a university teacher's planned approach to student learning will determine their actual practice in the classroom ${ }^{[18,19,21]}$. The interviewed nurse educators believed that the integration of identified culturally sensitive teaching strategies in their practice was a direct result of their engagement in the workshops, as noted in the comment:

Overall the workshop has given me a greater understanding of the Hong Kong operation and experiences and how to better accommodate international students by adapting my teaching style and practices. (Nurse Educator \#1)

The opportunity to work with colleagues to extrapolate and refine the identified strategies in small groups was acknowledged as a key factor in the integration of new and varied teaching approaches in practice. The nurse educators highlighted their strengthened confidence to implement new strategies in their practice resultant from a better understanding of the 'student experience' and 'teaching international students'. Academic staff teaching in offshore programs should be provided with staff development opportunities that challenge their stereotypes and Western

paradigms ${ }^{[2,18,22]}$. By developing academic knowledge of culture and skills to provide inclusive curriculum is an important aspect for improving staff and student experiences.

\section{Conclusion}

Using a reflective approach has been supported in this project as an important and valued means of professionally developing nurse educators. A core requirement of the reflective approach described in this paper, which could inform 
similar profession development initiatives, is the comprehensive and thoughtful consideration of the teaching context. This approach has enabled teachers in an offshore program to reconceptualise their practice and envisage how they can better meet the educational and cultural needs of international students. The teaching transformations that were identified and implemented were considered by the nurse educators involved in the project to have strengthened the delivery of subjects in the offshore program. Further research could seek to assess whether the teaching developments resulting from this initiative have had a positive bearing on student outcomes and experience. An integral focus of this future deliberation should prioritise the student perspective.

\section{References}

[1] Marginson S. The phenomenal rise of international degrees down under: Lucrative lessons of US Institutions? Change. 2004; 34(3): 34-44. http://dx.doi.org/10.1080/00091380209601854

[2] Jeong SYS, Hickey N, Levett-Jones T, Pitt V, Hoffman K., Norton CA. Understanding and enhancing the learning experiences of culturally and linguistically diverse nursing students in an Australian bachelor of nursing program. Nurse Education Today. 2011; 31: 238-244. PMid:21078536 http://dx.doi.org/10.1016/j.nedt.2010.10.016

[3] Feast V, Bretag T. Responding to crises in transnational education: New challenges for higher education. Higher Education: Research \& Development. 2005; 24(1): 63-78. http://dx.doi.org/10.1080/0729436052000318578

[4] $\mathrm{Xu} \mathrm{Y,} \mathrm{Davidhizar} \mathrm{R.} \mathrm{Intercultural} \mathrm{communication} \mathrm{in} \mathrm{nursing} \mathrm{education:} \mathrm{when} \mathrm{Asian} \mathrm{students} \mathrm{and} \mathrm{American} \mathrm{faculty} \mathrm{converge.}$ Journal of Nursing Education. 2005; 44(5): 209-15. PMid:15916024

[5] Amaro D, Abriam-Yago, K., Yoder, M. Perceived barriers for ethnically diverse students in nursing programs. Journal of Nursing Education. 2006; 45(7): 247-254. PMid:16863104

[6] Lee EL. Linguistic and cultural factors in East Asian students" oral participation in US university classrooms. International Education. 2007; 36 (2): 27-47.

[7] McBurnie G, Ziguras C. Transnational Education: Current issues and future trends in offshore Higher Education. London:Routledge Falmer. 2007.

[8] Dunn L, Wallace M. Australian academics and transnational teaching: an exploratory study of their preparedness and experiences. Higher Education Research \& Development. 2006; 25(4): 357-369. http://dx.doi.org/10.1080/07294360600947343

[9] Ruth-Sahd, LA. Reflective practice: a critical analysis of data-based studies and implications for nursing education. Journal of Nursing Education. 2003; 42 (11): 488-497. PMid:14626387

[10] O’Connor A, Hyde A. Teaching reflection to nursing students: a qualitative study in an Irish context. Innovations in Education and Teaching International. 2005; 42(4): 291-303. http://dx.doi.org/10.1080/14703290500291842

[11] Schön D. Educating the reflective practitioner. Jossey-Bass, San Francisco. 1987.

[12] Rolfe G, Freshwater D, Jasper M. Critical reflection for nursing and the helping professions, a user's guide. Palgrave Macmillan, Hampshire. 2001.

[13] Johns C. Becoming a Reflective practitioner 3rd Edition. Wiley-Blackwell UK. 2009.

[14] Colosimo R, Xu Y. Research on shame: implications for English as a second language nursing students. Home Health Care Management and Practice. 2006; 19(1): 72-75. http://dx.doi.org/10.1177/1084822306292226

[15] Green W. Write on or write off? An exploration of Asian international students approaches to essay writing at an Australian university. Higher Education Research \& Development. 2007. http://dx.doi.org/10.1080/07294360701494336

[16] Edgecombe K., Jennings M \& Bowden M. International nursing students and what impacts their clinical learning. Nurse Education Today. 2013; 33(2): 138-142. PMid:22939701 http://dx.doi.org/10.1016/j.nedt.2012.07.015

[17] Chong WH. The role of personal agency beliefs in academic self-regulation: an Asian perspective. School Psychology International. 2007; 28(1): 63-76. http://dx.doi.org/10.1177/0143034307075681

[18] Ryan GJ, Dogbey E. Seven strategies for international nursing student success: A review of the literature. Teaching and Learning in Nursing. 2012; 7: 103-107. http://dx.doi.org/10.1016/j.teln.2012.01.007

[19] Martin E, Prosser M, Trigwell K., Ramsden P, Benjamin J. What university teachers teach and how they teach it. Instructional Science. 2000; 28: 387-412. http://dx.doi.org/10.1023/A:1026559912774

[20] Reeves TC, Herrington J, Oliver R. Design research: A socially responsible approach to instructional technology research in higher education. Journal of Computing in Higher Education. 2005; 16(2): 96-115. http://dx.doi.org/10.1007/BF02961476

[21] Brookfield S. Critically Reflective Practice. Journal Continuing Education Health Professional. 2012; 4: 197-205.

[22] Crichton J, Paige M, Papademetre L \& Scarino A. Integrated resources for intercultural teaching and learning in the context of internationalization in higher education. Research Centre for Language and Cultures Education, University of South Australia. 2004. 